\title{
Nitrosourea Compound
}

National Cancer Institute

\section{Source}

National Cancer Institute. Nitrosourea Compound. NCI Thesaurus. Code C699.

Any of a class of alkylating agents that contain both a nitroso group and a urea. They are lipophilic and can cross the blood-brain barrier. 